ANNALES

POLONICI MATHEMATICI

$85.2(2005)$

\title{
On locally biholomorphic mappings from multi-connected onto simply connected domains
}

\author{
by Piotr Liczberski (Łódź) and \\ Victor V. Starkov (Petrozavodsk and Łódź)
}

\begin{abstract}
We continue E. Ligocka's investigations concerning the existence of $\mathrm{m}^{-}$ valent locally biholomorphic mappings from multi-connected onto simply connected domains. We decrease the constant $m$, and also give the minimum of $m$ in the case of mappings from a wide class of domains onto the complex plane $\mathbb{C}$.
\end{abstract}

1. Introduction. The Riemann theorem guarantees that any two simply connected domains of the plane $\mathbb{C}$, different from $\mathbb{C}$, are biholomorphically equivalent. In view of the Poincaré theorem (see e.g. [Go, Chap. VI, §1]) every simply connected domain $D \varsubsetneqq \mathbb{C}$ can be mapped locally biholomorphically onto every multi-connected domain $G$ with more than two boundary points, but usually in an infinitely valent way. On the other hand, if $G$ is a $k$-connected domain without isolated boundary points and $D \varsubsetneqq \mathbb{C}$ is a simply connected domain, then the Grunsky theorem (see e.g. [Go, Chap. VI, §5]) gives the existence of a mapping $f$ from $G$ onto $D$, which is holomorphic, $k$-valent, but not locally biholomorphic; in this case $k$-valence of $f$ means that for every $w \in D$ the equation $f(z)=w$ has in $G$ exactly $k$ solutions (counting multiplicity). Chapter VI of [Go] is, in particular, devoted to the study of similar problems.

E. Ligocka [Li] has found a class of multi-connected domains which can be mapped onto the unit disc $B(0,1)$ locally biholomorphically and $m$-valently, where $m \leq 24$ (in our paper, similarly to [Li], a mapping $f$ from $X$ onto $Y$ is called $m$-valent, $m \in \mathbb{N}$, if for each $y \in Y$ the set $\left\{f^{-1}(y)\right\}$ has no more than $m$ elements).

E. Ligocka has also proved that every finitely connected domain of $\mathbb{C}$, not biholomorphic to $\mathbb{C} \backslash\{0\}$, can be mapped onto $\mathbb{C}$ locally biholomorphically and $m$-valently, for some $m \in \mathbb{N}$.

2000 Mathematics Subject Classification: Primary 30C55; Secondary 30F99, 32H02.

Key words and phrases: locally biholomorphic mappings, finitely valent mappings, Riemann surfaces. 
In the present paper we prove that $m=3$ and $m=5$ in the case of locally biholomorphic mappings from domains of a much wider class onto $\mathbb{C}$ and onto $B(0,1)$, respectively. We also show that in the first case the constant $m=3$ is best possible.

2. Main results. We will consider domains with an isolated boundary fragment.

Definition. We shall say that a domain $D \subset \mathbb{C}$ has an isolated boundary fragment if one of the following conditions holds:

(I) There exists a continuum $K \subset \partial D$, different from a point, and an open set $U$ such that $K \subset U$ and $(\partial D \backslash K) \cap U=\emptyset$.

(II) There exists a Jordan arc $\Gamma \subset \partial D$ with distinct ends $\xi, \eta$ and an open disc $B$ such that $\xi, \eta \in \partial B, \Gamma \backslash\{\xi, \eta\} \subset B$ and $(\partial D \backslash \Gamma) \cap B=\emptyset$.

(III) There exists a point $a \in \partial D$ and an open disc $B(a)$ centred at $a$ such that $(B(a) \backslash\{a\}) \cap \partial D=\emptyset(a$ is an isolated point of the boundary $\partial D)$.

We will prove the following theorem:

THEOREM 1. If a domain $D \subset \mathbb{C}, D \neq \mathbb{C} \backslash\{a\}$, has an isolated boundary fragment, then there exists a 3-valent locally biholomorphic mapping from $D$ onto $\mathbb{C}$. The constant 3 is best possible.

In the proof we will consider three cases, according to the cases in the above definition. In the case of a domain with an isolated boundary fragment of type (III) we use the following lemma.

Lemma. Let $D \subset \mathbb{C}$ be a domain, infinitely connected or $k$-connected, $k>2$, such that $\partial D=E \cup \infty$, where $E$ is a bounded set. Then there exist two points of $E$ such that $E$ lies on one side of the straight line through those points.

Proof. Let $K$ be the closed convex hull of $E$ and let $l_{z}$ be a support straight line to the set $K$ at the point $z \in \partial K$.

First we will show that there exists $z \in \partial K$ such that $l_{z} \cap K \neq\{z\}$. Suppose, on the contrary, that $l_{z} \cap K=\{z\}$ for every $z \in \partial K$. Fix $z \in \partial K$ and denote by $l_{n}, n \in \mathbb{N}$, the straight lines parallel to $l_{z}$, at distance $1 / n$ from $l_{z}$ and lying on the same side of $l_{z}$ as $K$. Taking, for every $n \in \mathbb{N}$, a point $z_{n} \in E$ lying between $l_{n}$ and $l_{z}$, we obtain a sequence $\left(z_{n}\right)$ which has a subsequence convergent to a point $a \in E$, because $E$ is a compact set. Of course, $a \in l_{z}$. Since $l_{z} \cap K=\{z\}$, we have $a=z$. Thus, $z \in E$ and so $\partial K \subset E$, by the arbitrariness of $z$.

Since $\partial K$ is a curve which bounds a convex compact set, we deduce that $\partial D=\partial K \cup \infty$. Thus, $D$ is a two-connected domain, which contradicts the assumptions of the lemma. 
Now, let $z \in \partial K$ be such that $l_{z} \cap K \neq\{z\}$. Then in $l_{z} \cap K$, there exist points different from $z$, lying on one side of $z$. Among them there exists a point $z^{\prime}$ most distant from $z$. Similarly, we choose a point $z^{\prime \prime}$ most distant from $z$ among all points lying on the other side of $z$ (if such points do not exist, then we take $z^{\prime \prime}=z$ ). From the choice of $z^{\prime}, z^{\prime \prime}$ and from the properties of the closed convex hull $K$ of $E$ it follows that $z^{\prime}, z^{\prime \prime} \in E$.

Hence, the assertion of the lemma holds.

Now, we give the proof of Theorem 1 .

Proof. 1. First, assume that $D$ has an isolated boundary fragment of type (III). Then, by a homography, we map the isolated point $a$ of $\partial D$ onto $\infty$, and the rest of $\partial D$ onto a compact set $E$.

In view of the lemma there exist two different points in $E$ such that $E$ lies on one side of the straight line going through those points. Take two points of the intersection of $E$ and this line such that their distance is maximum possible, and send them onto $-1,1$ by a linear mapping. We can assume that then the set $E$ transforms onto a compact set included in the upper half-plane $P^{+}=\{z \in \mathbb{C}: \operatorname{Im} z>0\}$. As a result, $D$ is mapped biholomorphically onto a domain $D_{1}$ which includes the lower half-plane $P^{-}=\{z \in \mathbb{C}: \operatorname{Im} z<0\}$, the rays $(-\infty,-1),(1, \infty)$, but does not include the points $-1,1$.

The polynomial $Q(z)=z^{3}-3 z$ is locally biholomorphic in $D_{1}$. We will show that $Q\left(D_{1}\right)=\mathbb{C}$.

Indeed, since $S=(-\infty,-1) \cup(1, \infty) \subset D_{1}$ and $Q(S)=\mathbb{R}$, it is sufficient to observe that the equation $Q(z)=w, w \in \mathbb{C} \backslash \mathbb{R}$, has a solution $z \in P^{-}$. This follows from the fact that the roots $\zeta_{1}, \zeta_{2}, \zeta_{3}$ of this equation belong to $\mathbb{C} \backslash \mathbb{R}$ and $\zeta_{1}+\zeta_{2}+\zeta_{3}=0$.

2. Now consider $D$ with an isolated boundary fragment of type (II). We can assume that $(\partial D \backslash \Gamma) \cap \partial B=\emptyset$.

Let $G$ be a subdomain of $D$ which is included between the Jordan arc $\Gamma$ and an open $\operatorname{arc} \gamma$ of the circle $\partial B$, joining the ends of $\Gamma$ (see the definition).

Denote by $K$ the connected component of the boundary $\partial D$ such that $\Gamma \subset K$ and by $\widehat{D}$ the simply connected domain such that $D \subset \widehat{D}, \partial \widehat{D}=K$. In view of the Riemann theorem there exists a biholomorphic mapping from $\widehat{D}$ onto the half-plane $P^{+}$. Denote by $D_{*}, G_{*}, \gamma_{*}$ the images of the sets $D, G, \gamma$, respectively under the above mapping.

Since $\partial G=\gamma \cup \Gamma$ is a Jordan curve, all points of $\partial G$ are attainable from $G$. All points of $\gamma_{*}$ are attainable from $G_{*}$, and $\gamma_{*}$ is the homeomorphic image of $\gamma$. Since the set of points which correspond to points of $\partial G_{*}$ attainable from $G_{*}$ is everywhere dense in $\gamma \cup \Gamma$ (see [Go, Chap. II, §3]), there exist at least two points $\zeta_{1}, \zeta_{2} \in \partial G_{*} \backslash \gamma_{*}$ attainable from $G_{*}$. From this it follows that $\zeta_{1}, \zeta_{2} \in \mathbb{R}$. 
Let $l_{1}, l_{2} \subset G_{*}$ be the two Jordan arcs which end in $\zeta_{1}, \zeta_{2}$, and let $\gamma_{1}, \gamma_{2}$ be the preimages in $G$ of $l_{1}, l_{2}$, respectively. Denote by $\gamma_{3} \subset G$ a Jordan arc which joins the arcs $\gamma_{1}, \gamma_{2}$, and by $l_{3} \subset G_{*}$ the image of $\gamma_{3}$. Of course, we can choose $\gamma_{3}$ in such a way that the set $\gamma_{1} \cup \gamma_{2} \cup \gamma_{3} \cup \Gamma$ bounds a simply connected domain $G^{\prime} \subset G$ and $\partial G^{\prime}$ is a Jordan curve. Then the closure of $G^{\prime}$ is homeomorphic to the closure of the domain $G_{*}^{\prime} \subset G_{*}$, bounded by the curve $l_{1} \cup l_{2} \cup l_{3} \cup \mathbb{R}$.

Therefore, in $G_{*}^{\prime}$ there exists a semi-disc with centre $z_{0} \in \mathbb{R}$ and with diameter included in $\mathbb{R}$.

Applying the function $\left(z_{0}-z\right)^{-1}$ we map $D_{*}$ biholomorphically onto a domain $D_{1} \subset P^{+}$such that $\partial D_{1} \backslash \mathbb{R}$ is included in a disc centred at zero, of a sufficiently large radius.

By a translation we transform the upper half-plane $P^{+}$onto itself in such a way that $\partial D_{1} \backslash \mathbb{R}$ is included in the sector $\{z: \operatorname{Arg} z \in(0, \pi / 4)\}$.

To this translation image of $D_{1}$ we now apply the mapping $1+e^{i \pi \alpha} z^{1-\alpha}$, where $\alpha \in(0,1 / 4)$ and $z^{1-\alpha}$ is the main branch of the power. Then $D_{1}$ maps onto a domain $D_{2} \subset\{z: \operatorname{Arg}(z-1) \in(\pi \alpha, \pi)\}$, whose boundary $\partial D_{2}$ consists of rays $(-\infty, 1],\left\{1+t e^{i \pi \alpha}: t \geq 0\right\}$ and maybe of other components lying in the sector $\left\{z: \operatorname{Arg}(z-1) \in\left(\pi \alpha, \frac{\pi}{4}(1+3 \alpha)\right)\right\}$.

Next, by the mapping $\varphi(z)=z^{2}$, we transform $D_{2}$ biholomorphically onto $D_{3}=\varphi\left(D_{2}\right)$. The boundary $\partial D_{3}$ consists of the ray $[0, \infty)$, of the $\operatorname{arc} L=\left\{\left(1+t e^{i \pi \alpha}\right)^{2}: t \geq 0\right\}$ and maybe of other components, which are included in $P^{+}$. Observe that $L$ lies in the sector $\{z: \operatorname{Arg} z \in(0,2 \pi \alpha)\}$ and is asymptotic to the ray $\left\{t e^{i 2 \pi \alpha}: t \geq 0\right\}$ as $t \rightarrow \infty$.

Let $\psi(z)=(z-1)^{1+\alpha / 2}=\exp \{(1+\alpha / 2)[\ln |z-1|+i \operatorname{Arg}(z-1)]\}$, where $\operatorname{Arg}(z-1)$ varies in $(0,2 \pi)$, and let $D_{4}$ be the biholomorphic image of $D_{3}$ under $\psi$.

Rotation through $-\pi \alpha / 2$ sends $D_{4}$ onto a domain $D_{5}$ such that $D_{5} \supset P^{-}$ and $\partial D_{5} \supset[-1,0] \cup\{z: \operatorname{Arg} z=\pi \alpha / 2\} \cup L^{\prime}$, where $L^{\prime}$ is the image of the arc $L$ under the mapping $e^{-i \pi \alpha / 2} \psi(z)$; the boundary $\partial D_{5}$ may also include some points of $P^{+}$. Therefore, applying successively the mappings $1+2 z$ and $Q(z)$ to $D_{5}$, we obtain a locally biholomorphic and not more than 3 -valent mapping from $D_{5}$ onto $\mathbb{C}$, because $Q\left(P^{-}\right) \supset \mathbb{C} \backslash \mathbb{R}$ and $Q((-\infty,-1) \cup(1, \infty))=\mathbb{R}$, as shown in the first part of the proof.

As a result, we have a locally biholomorphic 3-valent mapping from $D$ onto $\mathbb{C}$.

3. Now let $D$ have an isolated boundary fragment of type (I). Then $K$ is the boundary of a simply connected domain $D_{0}$ such that $D \subset D_{0}$ and $\partial D \backslash K \subset K_{0}$, where $K_{0} \subset D_{0}$ is a compact set in $\overline{\mathbb{C}}$. Map $D_{0}$ biholomorphically onto the upper half-plane $P^{+}$. Then $K_{0}$ transforms onto a compact $K^{0} \subset P^{+}$. The image of $D$ under this mapping will be denoted by $D_{1}$. 
Now it is sufficient to transform $D_{1}$ as in the second part of the proof. Consequently, we obtain a locally biholomorphic and 3-valent mapping $f$ from $D$ onto $\mathbb{C}$.

4. Finally, we will prove that in our class of domains with an isolated boundary fragment, there exist domains which cannot be mapped onto $\mathbb{C}$ locally biholomorphically and 2-valently.

Set $D=\mathbb{C} \backslash\left\{a_{1}, \ldots, a_{M}\right\}$, where $M>1$ is an integer, and suppose that there exists a locally biholomorphic 2 -valent mapping $f$ from $D$ onto $\mathbb{C}$.

Of course, the points $a_{1}, \ldots, a_{M}$ and $\infty$ are isolated singularities of $f$. The Picard theorem and our supposition that $f$ is 2 -valent imply that none of the above points can be an essential singularity of $f$. Thus $f$ is rational as a meromorphic function on $\overline{\mathbb{C}}$. From this and our supposition that $f$ is 2 -valent, it follows that $f$ has one pole of the second order at $\infty$, or one pole of the first order at $\infty$ and one pole of the first order at a point $\alpha \in \mathbb{C}$, or poles of the first order at two points $\alpha, \beta \in \mathbb{C}, \alpha \neq \beta$, or one pole of the second order at a point $\alpha \in \mathbb{C}$. Equivalently, $f$ is defined by one of the following formulas:

(i) $f(z)=A z^{2}+B z+C$,

(ii) $f(z)=A z+B+\frac{C}{z-\alpha}$,

(iii) $f(z)=A+\frac{B z+C}{(z-\alpha)(z-\beta)}$.

In case (i), if $A=0$, then $f$ has the form $f(z)=B z+C$ and it cannot map $D$ onto $\mathbb{C}$. Assume that $A \neq 0$; we can put $A=1$. If we write $w_{0}=f\left(z_{0}\right)$, where $z_{0}=-B / 2$, then $z_{0} \notin D$, because $f$ is locally biholomorphic in $D$ and $f^{\prime}\left(z_{0}\right)=0$. Since $f(z)=\left(z-z_{0}\right)^{2}+w_{0}$, the equation $f(z)=w_{0}$ has only the double root $z_{0}$. The assumption that $f$ is 2 -valent and the fact that $z_{0} \notin D$ show that $w_{0} \notin f(D)$. Hence $f(D) \neq \mathbb{C}$.

In case (ii) we can rewrite $f$ in the form $f(z)=A(z-\alpha)+C /(z-\alpha)$. If $A=0$ or $C=0$, then $f$ is a homography and consequently $f(D) \neq \mathbb{C}$. Suppose $A C \neq 0$ and let $d,-d$ be the square roots of $C / A$. Then the points $z_{1}=\alpha+d$ and $z_{2}=\alpha-d$ do not belong to $D$, because $f$ is locally biholomorphic in $D$ and $f^{\prime}\left(z_{j}\right)=0, j=1,2$. Set $w_{j}=f\left(z_{j}\right), j=1,2$, and consider the equation $f(z)=w_{1}$. By the change of variable $\zeta=(z-\alpha) / d$ we get the equation $\zeta+1 / \zeta=2$, which has only the double root $\zeta=1$. Thus the equation $f(z)=w_{1}$ has the double root $z_{1}=\alpha+d$. Similarly, the equation $f(z)=w_{2}$ has the double root $z_{2}=\alpha-d$. This and the assumption that $f$ is 2 -valent shows that $w_{j} \notin f(D), j=1,2$. Hence $f(D) \neq \mathbb{C}$.

In case (iii) it suffices to consider the function $f(z)=\frac{B z+C}{(z-\alpha)(z-\beta)}$. Since $f^{\prime}(z) \neq 0$ in $D$, our supposition that $f$ is 2-valent implies that $g(z)=1 / f(z)$ is locally biholomorphic in $D$ and maps $D$ onto $\overline{\mathbb{C}} \backslash\{0\} 2$-valently. However, this is impossible, as shown in the previous cases. 
Since finitely connected domains, different from $\mathbb{C} \backslash\{0\}$, are domains with an isolated boundary fragment, we obtain the following generalization of Ligocka's result cited in the introduction.

Corollary 1. If $D \subset \mathbb{C}$ is a finitely connected domain, different from $\mathbb{C} \backslash\{0\}$, then there exists a locally biholomorphic and 3-valent mapping from $D$ onto $\mathbb{C}$. The constant 3 is best possible.

In [Li] it is also proved that there exists a function which maps, locally biholomorphically and 24-valently, a domain $D$ with an isolated component of $\overline{\mathbb{C}} \backslash D$ onto the open unit disc $B(0,1)$.

We will prove the following theorem.

THEOREM 2. If $D \subset \mathbb{C}$ is a multi-connected domain with an isolated boundary fragment of type (I) or type (II), then there exists a 5-valent, locally biholomorphic mapping from $D$ onto the disc $B(0,1)$.

Proof. 1. Asume that $D$ has an isolated boundary fragment of type (I) and let $K$ be the relevant continuum. Then $K$ is the boundary of a simply connected domain $D_{0} \subset \overline{\mathbb{C}}$ such that $D \cap D_{0} \neq \emptyset$. From the definition it follows that $\partial D \backslash K \subset K_{0}$, where $K_{0} \subset D_{0}$ is a compact set in $\overline{\mathbb{C}}$.

Let $\zeta=\varphi(z)$ be a biholomorphic mapping from $D_{0}$ onto $B(0,1)$ and let $B^{\prime}$ be a circular open neighbourhood of the point $\zeta=-1$. We can assume that the compact set $K^{0}=\varphi\left(K_{0}\right)$ is included in a simply connected domain $E_{1} \subset B(0,1)$ such that $E_{1} \cap B^{\prime} \neq \emptyset$.

Let $L=P^{+} \cap Q^{-1}((-\infty,-2))$, where $Q(\zeta)=\zeta^{3}-3 \zeta$. It is clear that $L$ is a simple and analytic arc which joins the points $\zeta=1, \zeta=\infty$. Fix $A \in L$ and denote by $D^{\prime}$ the simply connected domain which is obtained from $P^{+}$by removing the part of $L$ joining $A$ and $\infty$. Let $\eta=\psi(w)$ be a biholomorphic mapping from $D^{\prime}$ onto $B(0,1)$. Set $E_{2}=\psi\left(D^{\prime \prime}\right)$, where $D^{\prime \prime} \subset D^{\prime}$ is the domain bounded by the arc $L$ and the ray $[1,+\infty)$. The simply connected domain $E_{2}$ is bounded by an open arc of the circle $\partial B(0,1)$ and by a Jordan curve $\Gamma^{\prime \prime} \subset \overline{B(0,1)}$. We can assume that the above arc of $\partial B(0,1)$ includes $\zeta=1$. Then there exists a circular open neighbourhood $B_{1}$ of $\zeta=1$ such that $B_{1} \cap B(0,1)=B_{1} \cap E_{2}$.

The mapping $\frac{\zeta+a}{1+a \zeta}$, with properly chosen $a \in(-1,1)$, transforms $B(0,1) \backslash \overline{B^{\prime}}$ into $B(0,1) \cap B_{1}$. Then

$$
w=\psi^{-1}\left(\frac{\varphi(\zeta)+a}{1+a \varphi(\zeta)}\right)
$$

maps $D$ biholomorphically onto a domain $D_{2} \subset D^{\prime}$ such that $\partial D_{2} \backslash \partial D^{\prime} \subset$ $\psi^{-1}\left(E_{2}\right)=D^{\prime \prime}$.

Observe that $Q\left(P^{+}\right) \supset \mathbb{C} \backslash \mathbb{R}$ (just as for $P^{-}$in part 2 of the proof of Theorem 1). Moreover, $Q$ maps the set $(-\infty,-2) \cup(2, \infty)$ biholomor- 
phically onto itself and for every $w \in[-2,2]$ the equation $Q(z)=w$ has three roots (counting multiplicity). Thus $Q\left(P^{+}\right)=\mathbb{C} \backslash[-2,2]$ and $Q\left(D^{\prime}\right)=$ $\mathbb{C} \backslash\left([-2,2] \cup\left(-\infty, A^{\prime}\right]\right)$, where $A^{\prime}=Q(A)<-2$. Set $D_{3}=Q\left(D^{\prime}\right)$.

We will show that the mapping $Q$ in the upper half-plane $P^{+}$gives a 2-valent covering of $P^{+}$. To do this take $w \in P^{+}$and $R$ so large that all roots $z \in P^{+}$of the equation $Q(z)=w$ belong to the interior of the closed curve $\Gamma_{R}$ consisting of a semi-circle $C_{R}$ and the segment $[-R, R]$; denote by $N_{w}$ the number of these roots, counted with multiplicity. Using the argument principle, we have

$$
\begin{aligned}
2 \pi N_{w} & =\Delta_{\Gamma_{R}} \arg (Q(z)-w) \\
& =\Delta_{C_{R}} \arg \left(z^{3}\right)+\Delta_{C_{R}} \arg \left(1-\frac{3}{z^{2}}-\frac{w}{z^{3}}\right)+\Delta_{[-R, R]} \arg \left(z^{3}-3 z-w\right),
\end{aligned}
$$

hence, for $R \rightarrow \infty$,

$$
2 \pi N_{w}=3 \pi+\pi+o(1)
$$

Thus $N_{w}=2$.

Now observe that $Q\left(D^{\prime \prime}\right)=P^{+}$. Therefore $Q\left(D_{2}\right)=D_{3}$ and the mapping $Q$ in $D_{2}$ is locally biholomorphic and 2-valent.

In this way we obtain a locally biholomorphic and 2-valent mapping from $D$ onto $D_{3}$, but only points from $P^{+}$can be covered twice.

2 . Now we will show that in the case of $D$ with an isolated boundary fragment of type (II), there also exists a locally biholomorphic 2-valent mapping from $D$ onto $D_{3}$.

For this purpose, we map $D$ onto $D_{1}$ locally biholomorphically and 2valently (notation from part 2 of the proof of Theorem 1 ). Next, as in part 1 of the proof of Theorem 2 , we map $P^{+}$onto $B(0,1)$ by a homography such that the inner components of the image of $D_{1}$ belong to the domain $E_{1} \subset B(0,1)$. Now, to obtain a locally biholomorphic 2 -valent mapping from $D$ onto $D_{3}$, it is sufficient to repeat the same steps as in part 1 of the proof of Theorem 2 .

3 . Now we construct a locally biholomorphic 3-valent mapping from $D_{3}$ onto $B(0,1)$.

Let $0<c<d$ and transform $D_{3}$ onto $\mathbb{C} \backslash((-\infty, 0] \cup[c, d])$ linearly; then, of course, the upper half-plane $P^{+}$maps onto itself. Next applying the function $-i \frac{\sqrt{z}-1}{\sqrt{z}+1}(\sqrt{z}$ is the main branch of the square root) we transform $\mathbb{C} \backslash((-\infty, 0] \cup[c, d])$ onto $B(0,1)$ with a segment $[a i, b i]$ removed (in view of the arbitrariness of $c, d$, we can assume that $a, b$ are arbitrary numbers such that $-1<a<b<1$ ). This mapping sends $P^{+}$onto the right semi-disc.

Denote by $D_{4}$ the image of $B(0,1) \backslash[a i, b i]$ under the mapping $i \sqrt[4]{3} \frac{1+z}{1-z}$. Then $D_{4}=P^{+} \backslash l$, where $l$ is a closed arc included in the upper semicircle $\left\{z \in P^{+}:|z|=\sqrt[4]{3}\right\}$. Moreover, the right semi-disc of $B(0,1)$ will be transformed onto $D_{4}^{\prime}=\left\{z \in P^{+}:|z|>\sqrt[4]{3}\right\}$. In this way we obtain a locally 
biholomorphic and 2-valent mapping from $D$ onto $D_{4}$, but in $D_{4}$ only points from $D_{4}^{\prime}$ can be covered twice.

In view of the arbitrariness of $a, b$ we can assume that the arc $l$ starts from the point $\sqrt[4]{3} e^{i \pi / 4}$ and finishes at $\sqrt[4]{3} e^{3 i \pi / 4}$.

Now consider the image of $D_{4}$ under the mapping

$$
f(z)=\frac{z^{3}-3 z}{z^{2}-1} .
$$

Since $f(z)=z+\frac{z}{z+1}+\frac{z}{z-1}$ and every summand of the right-hand side transforms $P^{+}$onto itself and $f(\mathbb{R})$ is the real axis, covered three times, it follows that $f\left(P^{+}\right)$is the upper half-plane $P^{+}$covered three times. In the remaining part of the proof we will treat the image $f(X)$ of a domain $X \subset D_{4}$ as a part of the Riemann surface of the inverse function.

Observe that $f^{\prime}$ vanishes in $P^{+}$only at the points $z_{1}=\sqrt[4]{3} e^{i \pi / 4}, z_{2}=$ $\sqrt[4]{3} e^{3 i \pi / 4}$. They are the branch points of the Riemann surface $f\left(P^{+}\right)$. It is obvious that $f(-\sqrt[4]{3})=\sqrt[4]{3^{3}}, f(\sqrt[4]{3})=-\sqrt[4]{3^{3}}$ and

$$
f:\left\{\sqrt[4]{3} e^{i t}: t \in[0, \pi]\right\} \rightarrow\left\{\sqrt[4]{3^{3}} e^{i \tau}: \tau \in[\pi, 0]\right\} .
$$

We will examine the image $f\left(\partial D_{4}^{\prime}\right)$. We have:

$$
\begin{aligned}
f:(-\infty,-\sqrt[4]{3}] & \rightarrow\left(-\infty, \sqrt[4]{3^{3}}\right] \\
f:\left\{\sqrt[4]{3} e^{i t}: t \in[\pi, 3 \pi / 4]\right\} & \rightarrow\left\{\sqrt[4]{3^{3}} e^{i \tau}: \tau \in[0,7 \pi / 12]\right\},
\end{aligned}
$$

and since $z_{1}$ and $z_{2}$ are simple zeros of $f^{\prime}(z)$, we also have:

$$
\begin{aligned}
f:\left\{\sqrt[4]{3} e^{i t}: t \in[3 \pi / 4, \pi / 4]\right\} & \rightarrow\left\{\sqrt[4]{3^{3}} e^{i \tau}: \tau \in[7 \pi / 12,5 \pi / 12]\right\}, \\
f:\left\{\sqrt[4]{3} e^{i t}: t \in[\pi / 4,0]\right\} & \rightarrow\left\{\sqrt[4]{3^{3}} e^{i \tau}: \tau \in[5 \pi / 12, \pi]\right\}, \\
f:(\sqrt[4]{3},+\infty] & \rightarrow\left[\sqrt[4]{3^{3}},+\infty\right] .
\end{aligned}
$$

From this it follows that $f\left(D_{4}^{\prime}\right)$ is a Riemann surface $\mathfrak{R}_{1}$ which consists of two copies of the semi-disc $B^{+}=\left\{z \in P^{+}:|z|<\sqrt[4]{3^{3}}\right\}$ and one copy of the domain $\widehat{B^{+}}=\left\{z \in P^{+}:|z|>\sqrt[4]{3^{3}}\right\}$. Moreover, $\widehat{B^{+}}$is stuck to one copy of $B^{+}$along the arc $\left\{\sqrt[4]{3^{3}} e^{i \tau}: \tau \in[7 \pi / 12, \pi]\right\}$, but to the other copy of $B^{+}$along the arc $\left\{\sqrt[4]{3^{3}} e^{i \tau}: \tau \in[0,5 \pi / 12]\right\}$. Accordingly, the image of the semi-disc $\left\{z \in P^{+}:|z|<\sqrt[4]{3}\right\}$ is a Riemann surface $\mathfrak{R}_{2}$ which consists of two copies of $\widehat{B^{+}}$and one copy of $B^{+}$, stuck along arcs of the semi-circle $\left\{z \in P^{+}:|z|=\sqrt[4]{3^{3}}\right\}$.

Therefore, the Riemann surface $\mathfrak{R}=f\left(D_{4}\right)$ covers the whole upper halfplane $P^{+}$, because $f(l)=\left\{\sqrt[4]{3^{3}} e^{i \tau}: \tau \in[5 \pi / 12,7 \pi / 12]\right\} \subset f\left(\left\{\sqrt[4]{3} e^{i t}:\right.\right.$ $t \in[0, \pi / 4)\}) \cup f\left(\left\{\sqrt[4]{3} e^{i t}: t \in(3 \pi / 4, \pi]\right\}\right)$. Moreover, the mapping $f$ is not more than 2-valent in $D_{4}^{\prime}$ and in $D_{4} \backslash D_{4}^{\prime}$. 
However, the points from $D_{4} \backslash D_{4}^{\prime}$ were univalently covered by the locally biholomorphic image of the domain $D$, and the points $D_{4}^{\prime}$ were covered not more than twice. Thus, the points of the Riemann surfaces $\mathfrak{R}_{2}$ are covered not more than twice by the locally biholomorphic image of $D$, and the points of $\mathfrak{R} \backslash \mathfrak{R}_{2}$ are covered univalently.

From this it follows that the constructed mapping on $D$ covers the upper half-plane $P^{+}$not more than fivefold.

Now it is sufficient to map $P^{+}$biholomorphically onto $B(0,1)$.

Conjecture. The constant 5 from Theorem 2 can be replaced by 3 .

E. Ligocka $[\mathrm{Li}]$ has remarked that theorems about locally biholomorphic mappings from multi-connected domains onto a disc can be applied in the investigation of mappings from domains of the space $\mathbb{C}^{n}, n \geq 1$, onto $n$ dimensional complex manifolds.

Observe that if we combine our Theorem 2 with Fornæss and Stout's result from [FS], we obtain the following corollaries:

Corollary 2. If a multi-connected domain $D \subset \mathbb{C}$ has an isolated boundary fragment of type (I) or type (II) and $X$ is a connected Riemann surface (compact or open), then there exists an m-valent locally biholomorphic mapping $f$ from $D$ onto $X$ with $m \leq 5 \cdot 14=70$.

Corollary 3. If $X=D_{1} \times \cdots \times D_{n}$, where the domains $D_{j}, j=$ $1, \ldots, n$, fulfil the assumptions of Corollary 2 , and $Y$ is a connected paracompact $n$-dimensional complex manifold, then there exists a locally biholomorphic and $m$-valent mapping $f$ from $X$ onto $Y$ with $m \leq 5^{n}\left[(2 n+1) 4^{n}+2\right]$.

\section{References}

[FS] J. E. Fornæss and E. L. Stout, Spreading polydiscs on complex manifolds, Amer. J. Math. 99 (1977), 933-960.

[Go] G. M. Goluzin, Geometric Theory of Functions of a Complex Variable, 2nd ed., Nauka, Moscow, 1966 (in Russian).

[Li] E. Ligocka, On locally biholomorphic surjective mappings, Ann. Polon. Math. 82 (2003), 127-135.

Institute of Mathematics

Technical University of Łódź

Żwirki 36

90-924 Łódź, Poland

E-mail: piliczb@p.lodz.pl

vstar@p.lodz.pl
Faculty of Mathematics Petrozavodsk State University

Pr. Lenina 33 185910 Petrozavodsk, Russia E-mail: vstar@psu.karelia.ru 